\title{
RouteFinder: Real-time Optimum Vehicle Routing using Mobile Phone Network
}

\author{
Najiba Ahmadullah, Shahpar Islam and Tarem Ahmed \\ Department of Electrical and Electronic Engineering \\ BRAC University \\ Dhaka, Bangladesh \\ Email: $\{$ u10310004, u10121052\}@ student.bracu.ac.bd, tarem@bracu.ac.bd
}

\begin{abstract}
Road traffic congestion is a major issue in most mega cities. The traffic jams are often exacerbated by drivers habitually following the same routes. A real-time, optimum vehicle routing system that takes traffic density into account can be a possible solution to this problem. This paper presents RouteFinder, a system of providing real-time traffic density mapping on the driver's smartphone using an in-vehicle module built using inexpensive components that communicates with the existing mobile telephone network, to enable the driver to choose the least congested route to a desired destination. The hardware module contains the essential elements of a cellular handset such as a SIM card. The location of the vehicle is determined through a standard triangulation algorithm performed using signals from the three nearest cellular base stations, and the location information is constantly updated at relevant Home Location Registers (HLRs) / Visitor Location Register (VLRs) at the telecom service provider using an intermediate MySQL database. An Android application developed for the driver's smartphone shows the present locations of all vehicles in all routes from the origin to the selected destination, with colour codes distinguishing between moving and stationary vehicles. We have implemented our device in 10 vehicles in Dhaka city. Our sample calculations have shown significant savings not only in terms of time, but also in fuel consumption.
\end{abstract}

\section{INTRODUCTION}

Publications from the Bangladesh Road Transport Authority (BRTA) show that 39 thousand new vehicles were registered in March 2014, making a total of around 2 million registered vehicles in the country [1]. This is the reason most commonly cited by road traffic management authorities in Bangladesh for perennial traffic congestions that plague many parts of this heavily-populated nation. On the other hand, official Government of Bangladesh publications claim over 20 thousand kilometres of urban roads and over 100 thousand kilometres of country roads as part of the national transportation network [2]. This indicates that there are often several routes to go from origin to destination. Traffic congestion is often created and exacerbated by people tending to follow the usual and well-traversed routes rather than taking a real-time decision to veer off the beaten path.

Traffic congestion in Asian metropolises like Bangkok, Kuala Lumpur, Jakarta and Manila can be so severe, that it may take up to an hour to reach a destination only a few kilometres away. This delay may become fatal if the vehicle is an ambulance or a fire brigade truck. Older vehicles heat up while being stuck in traffic jams, and the released exhaust fumes worsen the level of pollution and environmental damage.
In terms of economic loss, recent studies have put the number at 2.5 billion dollars annually as the deadweight loss to the economy in Bangladesh caused by traffic congestion [3].

A number of schemes have been recently proposed to track the course of a vehicle in real-time using existing cellular telephone networks. However, most researchers focus on tracking their own vehicle only, with the focus being security issues. We display the positions of all vehicles, with the objective of determining the optimum route to take to reach a destination accounting for traffic congestions along the way. We proposed a novel simple, inexpensive, elegant and lightweight system using signals from a Global System for Mobile Communications (GSM) cellular network. If each vehicle gets instantly connected to the network and notified in real-time about the traffic density in its path, then the time taken to reach any destination could be reduced. The vehicles could choose the route with the least delay and cost. In this paper we present RouteFinder, a system that uses standard communication signals from a mobile phone network and a digital map to determine the optimum route to reach a destination.

\section{A. Related Work}

There have recently been a lot of interest in developing inexpensive vehicle tracking systems using GSM networks with smartphone applications providing the front end user interface. The effort that comes closest to our work is the system proposed by Lee et al. [4]. Lee et al. have designed an in-vehicle device that uses a Global Positioning System (GPS) module to determine geographic coordinates of the vehicle at regular time intervals, and a General Packet Radio Service (GPRS) module to transmit and update the vehicle's location to a central database. A Google Maps ${ }^{T M}$ API is used to display the present location of the vehicle on a digital map in the driver's smartphone. The driver is subsequently provided with distance-to-go and time-remaining estimates to reach his desired destination. Our proposed system goes further in that the locations of all vehicles are displayed on the driver's smartphone application, which enables him to choose the leastcongested route. We have also used instead the triangulation method [5] to determine the coordinates of the vehicles, as is explained later in the paper.

Fuad and Drieberg have presented in [6] a remote vehicle tracking system using Short Messaging Services (SMS) updates sent from the vehicles using GSM modems to a central control tower. The SMS updates are then processed to display 


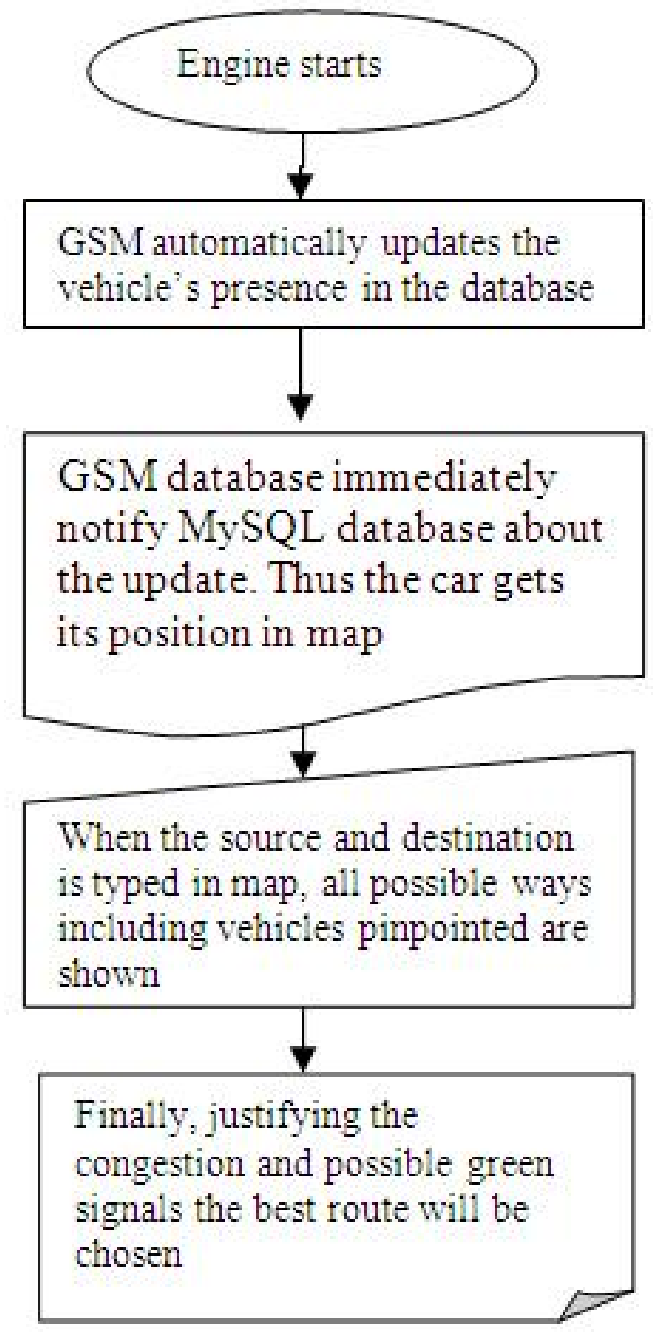

Fig. 1. Flow chart of proposed real-time vehicle routing system.

path of the vehicle on an online map. The objective of Fuad and Drieberg is to track stolen vehicles and to enable better fleet management for transportation and delivery companies. Chaturvedi and Srivastava have attempted to estimate road traffic characteristics through cellular ID updates made by vehicles carrying GSM modems [7]. They have also developed a new map-matching algorithm that can work in the midst of large location errors to estimate useful information such as origin-destination of a trip, route and duration of a trip. Gadri et al. [8] have presented a method for determining the location of a vehicle using an embedded GPS receiver and displaying the location on an Android ${ }^{T M}$-based digital map on a smartphone. Bajaj and Gupta [9] have suggested using Radio Frequency Identification (RFID) technology to transmit the location information, after determining it through an invehicle GPS receiver. It is clear that most researchers have restricted themselves to identifying their own locations, and displaying only their own positions on a digital map. The objective is to provide vehicle tracking for security purposes. We wish to provide the locations of all the vehicles on the route, and our objective is to determine the least-cost path to a driver's intended destination, accounting for traffic densities on the possible routes.

\section{B. Organization of Paper}

The rest of this paper is organized as follows. Section II presents our proposed RouteFinder real-time optimum vehicle routing system. Section III presents the results of our experiments. Section IV concludes and outlines the future potential of our approach.

\section{ROUteFinder Vehicle Routing System}

\section{A. System Outline}

In our proposed scheme, the car starts searching for, and registers with, the nearest Base Transceiver Station (BTS) [10] as soon as the ignition switch is turned on. This is achieved via a designated circuit containing a Subscriber Identity Module (SIM) and Network Interface Card (NIC) connected to the vehicle's electrical system. All essential functionalities of a cellular handset, formally known as a Mobile Station (MS), are also implemented in this hardware device, so that it is capable of performing essential activities such as registration and handoff.

The user interface available to the driver will contain a digital map such as Google Maps connected with an Android application that has been specifically developed for this purpose. A triangulation algorithm [5] is used to determine the coordinates of the vehicle. This is performed by using signal strength and Round Trip travel Time (RTT) observations from 3 BTSs in the vicinity of the vehicle. These readings are exchanged through natural call setup communication between an MS and nearby BTSs over Forward Control Channel / Reverse Control Channel (FCC/RCC) pairs. These measurements can be used to estimate 3 values for the radii and subsequently draw 3 circles with the BTSs at the centres as the possible loci of the vehicle. The intersection of the 3 circles will correspond with the actual present location of the said vehicle. This is then displayed on the digital map in the driver's smartphone.

The driver is then prompted to enter his destination address. Once this is done, the Android application will show the locations on the map of all the vehicles along possible routes to the destination, along with the locations of the traffic lights on those roads. A suitably weighted shortest-path algorithm [11] may then be used to compute the appropriate least-cost path.

A flow chart of our proposed scheme is shown in Fig. 1.

\section{B. Hardware Design}

Figure 2 shows the circuit diagram for the hardware components. $12 \mathrm{~V}$ DC power is used at the source, appropriately stepped down where necessary. A DC circuit filter is then used to reduce swell. Finally, a 7805 voltage regulator chip is used to further reduce the $12 \mathrm{~V} \mathrm{DC}$ to $5 \mathrm{~V} \mathrm{DC}$.

The NE555 timer is an 8 pin chip which is a wellestablished stable circuit. It is capable of working as a multivibrator as well as an accurate time-delay generator. It is used here as an astable circuit, also known as free running mode. It drives a Light Emitting Diode (LED) in the circuit which flashes on or off. We use 6 LEDs here that blink one 


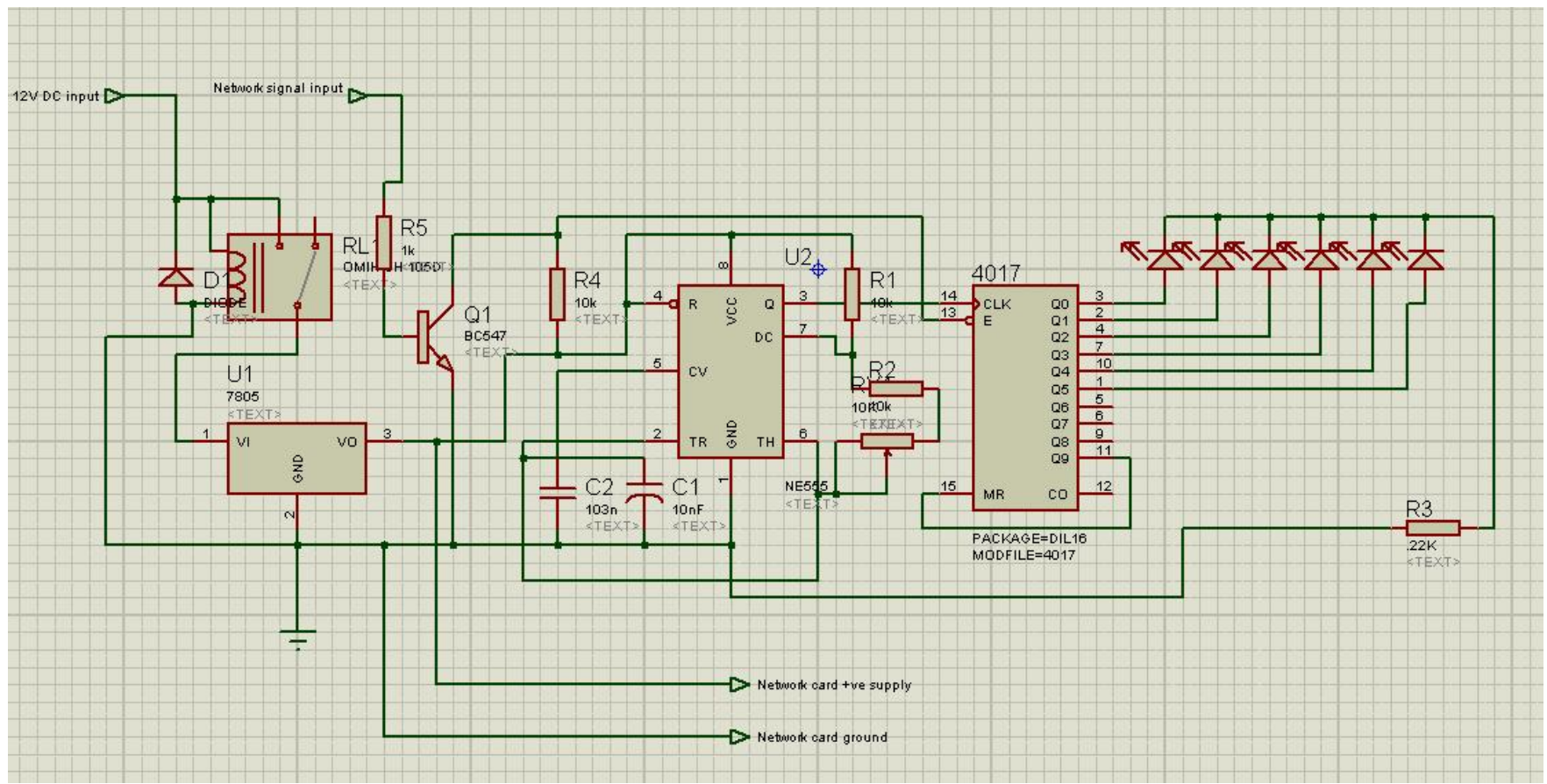

Fig. 2. Circuit diagram for the hardware components.

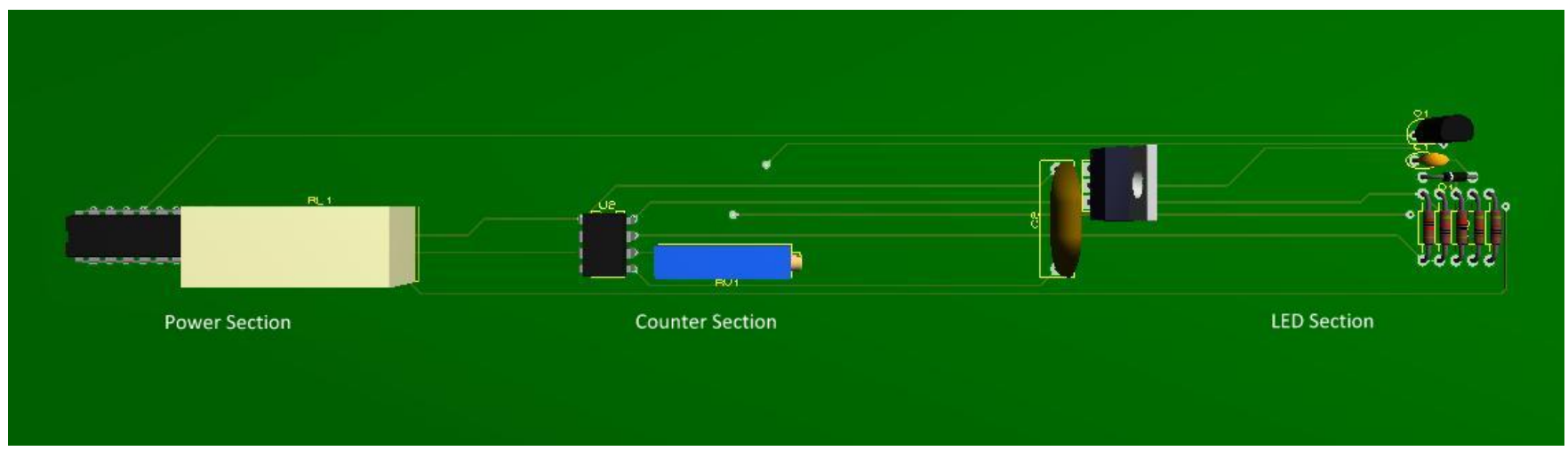

Fig. 3. Top-level three-dimensional snapshot of the hardware components.

after another and repeat the process to confirm connectivity with a BTS. This is so that the user is aware whenever the connection is lost. It also performs pulse generation, pulse position modulation and logic clock functionalities.

The electrically operated switch is connected to the ignition and used to control the turning on and off of the device. When it is turned on, the device establishes connection with the nearest BTS and the LEDs blink.

A decade counter is added to count the pulses. A 4017 chip is used in the timing circuit to switch the LEDs on and off. Moreover, the NE555 timer and decade counter are adjustable and comparatively simple to use. The decade counter is activated using pulses from the timer. The astable circuit pulses up the decade counter at regular intervals.

Figure 3 presents a top-level three-dimensional snapshot view of our device.

\section{Software Design}

We have developed an Android application that the driver will use on his smartphone. In the era of smartphones and and range of associated apps, we feel that this is the best choice for the operating user interface [12]. The Android application shows the positions of the other vehicles in the possible routes from the origin to the destination on Google Maps. The other vehicles are indicated on the map as Red dots if their positions are determined to be not changing with time (i.e. the cars are stuck in jam), and as Green dots if their positions are determined to be changing with time (i.e. they are moving). The coordinate information of every registered vehicle is refreshed after 10 seconds. Our future work will involve colour coding the degree (shade) of redness or greenness depending upon the rate of change of position of the vehicles' locations (i.e. their speeds).

The Home Location Register (HLR) and/or Visitor Location Register (VLR) databases [10] at the mobile telecommunication service provider will store the latitude and longitude co- 


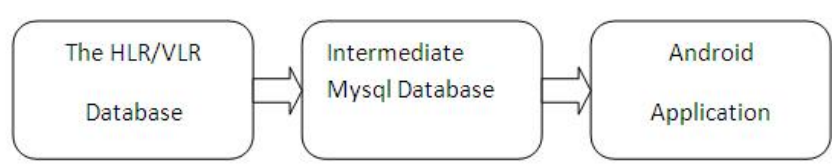

Fig. 4. Database relational architecture.

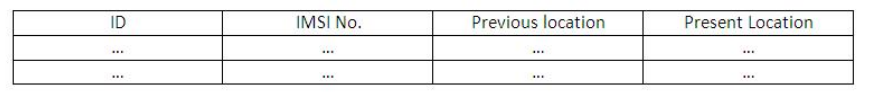

Fig. 5. Form of MySQL database table.

ordinates corresponding to every Mobile Station's International Mobile Subscriber Identity (IMSI) number. Figure 4 shows the architecture for the database relation. Oracle ${ }^{T M}$ is the most popular database software used by mobile network companies today. We connect a specific MySQL database [13] with the Oracle SQL database. The intermediate MySQL database provides the latitude and longitude information to the Android application. MySQL is mainly used as the database in websites and related applications, but not in the database systems of telecommunication companies. That is why we need to create a relation between them. A web-based administrative system called "phpMyAdmin" [14] is used to control and monitor the MySQL database. Phpmyadmin is easy to use and involves fewer implementations complexities compared to writing the database query language by hand. Android applications cannot fetch the data directly from MySQL database, but sends a string query called "Jason" to get the data [13]. We need to install a "Jason" plugin for phpMyAdmin to address this issue. The form of the MySQL database table is shown in Fig. 5.

We use Google API to connect the map with the Android application. This is a most convenient way of using Google Maps in an Android application. The Android version needed to run our application is Version 4.3 : Jelly Bean [15]. Figure 6 shows an example scenario of two possible routes between an origin-destination pair. It is observed that the relatively longer route has less traffic, indicating that it is expected to be faster.

\section{EXPERIMENTS}

\section{A. Results}

Our device was installed in 10 vehicles. The device is installed in such way that the network card will get the power needed through the car battery, regulated by a 7805 voltage regulator, when the vehicle's ignition switch is turned on.

When Driver 1 turns on his Android application, his coordinates are determined to be latitude 23.748485 degrees and longitude 90.403108 , and he sees the map presented as Fig. 7. Along with his present location, he is able to observe the density and speeds of the other vehicles around him. The Red arrows in Fig. 7 depict stationary vehicles while the Green arrows indicate vehicles that are moving.

Driver 1 now enters "Moghbazar" circle as his destination. He now sees the map presented as Fig. 8. He observes two possible routes of reaching his destination. A route that involves a slight detour is observed to contain Green arrows, whereas the direct road indicates many Red arrows.

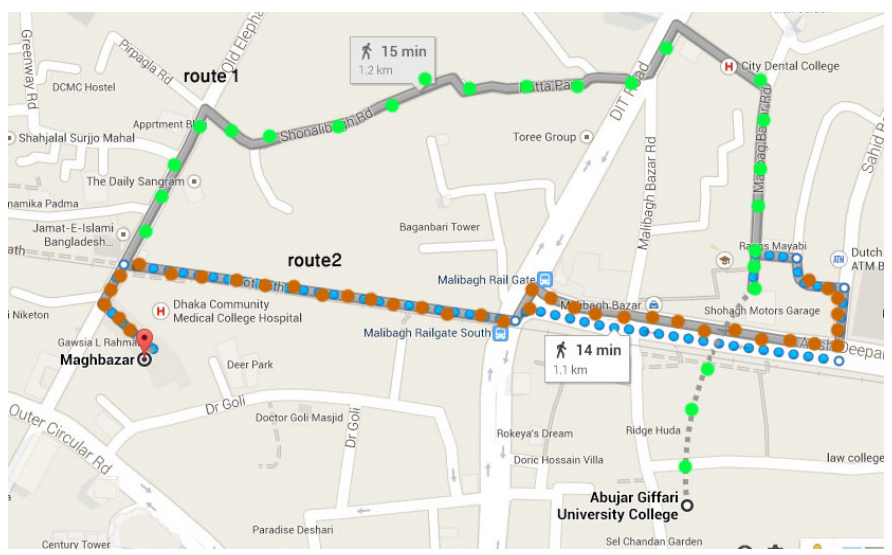

Fig. 6. Possible routes between two points with varying levels of traffic congestion. The relatively longer route is seen to have less traffic.

\section{B. Economic Analysis}

Here we do some sample calculations for estimated fuel consumptions. A typical passenger vehicle with a 1.3 litre engine consumes 1 litre of Octane approximately every 8 kilometres in urban areas and 12 kilometres in rural areas. If the vehicle runs on compressed natural gas (CNG), a common feature in many Asian countries including Bangladesh, it would go about 15 kilometres in urban areas and 20 kilometres in rural areas on 1 cubic metre of CNG. Our experiments have also indicated that if a vehicle remains stuck in traffic for about 30 minutes with its engine running, it will burn 1 cubic metre of CNG or 1 litre of Octane. We have assumed that the vehicle's air conditioner remains on throughout.

Suppose a driver chooses to go from "Mouchak" to "Bangla Motor" as shown on Fig. 9 and the direct route via "Moghbazar" is selected. The distance from "Mouchak" to "Moghbazar" is 750 metres while from "Moghbazar" to "Bangla Motor" is 1900 metres, making it a total distance of 2.65 kilometres. The estimated time needed to travel form "Mouchak" to "Moghbazar" is 3 minutes and from "Moghbazar" to "Bangla Motor" is 7 minutes, assuming typical speeds for Dhaka city roads. Furthermore, the traffic lights at "Moghbazar" and "Bangla Motor" intersections turn Green for 30 seconds after every 6 -minute interval.

In the route selected, given the characteristics of the traffic signals, the vehicle most likely has to wait for 6 minutes at "Moghbazar" intersection and another 6 minutes at "Bangla Motor" intersection. Total estimated travel time using this route is thus expected to be about $3+7+6+6=22$ minutes.

The vehicle will consume $2.65 / 15=0.1766$ cubic metre of CNG or $2.65 / 8=0.3313$ litre of Octane while it is moving, and $12 / 30=0.4$ cubic metre of $\mathrm{CNG}$ or $12 / 30=0.4$ litre of Octane while it is stationary at a traffic light. Total fuel consumption will be 0.57 cubic metre of $\mathrm{CNG}$ or 0.73 litre of Octane. At present rates of BDT 30 per cubic metre of CNG and BDT 98 per litre of Octane, the monetary costs of making this trip evaluate to BDT 17.10 in case of CNG and BDT 71.54 in case of Octane.

Now suppose that after using our RouteFinder application, the driver learns that a traffic jam is waiting ahead on his selected route, and he decides instead to take the alternate 


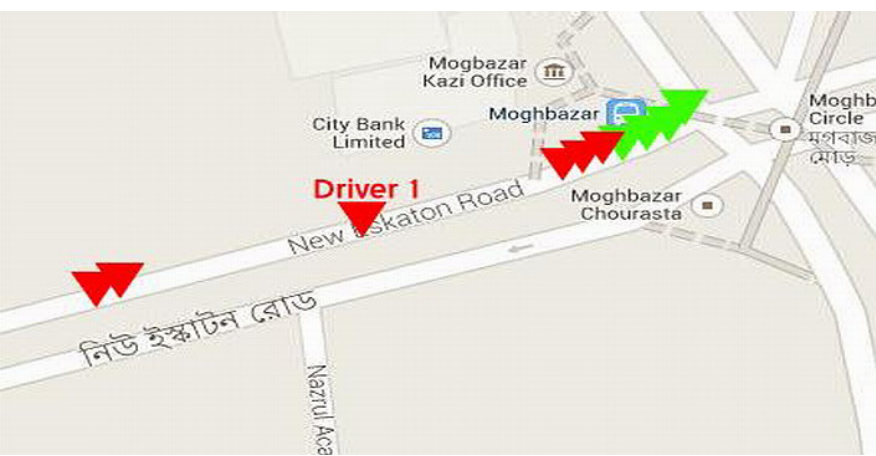

Fig. 7. View of Driver 1 immediately upon turning on car ignition switch.

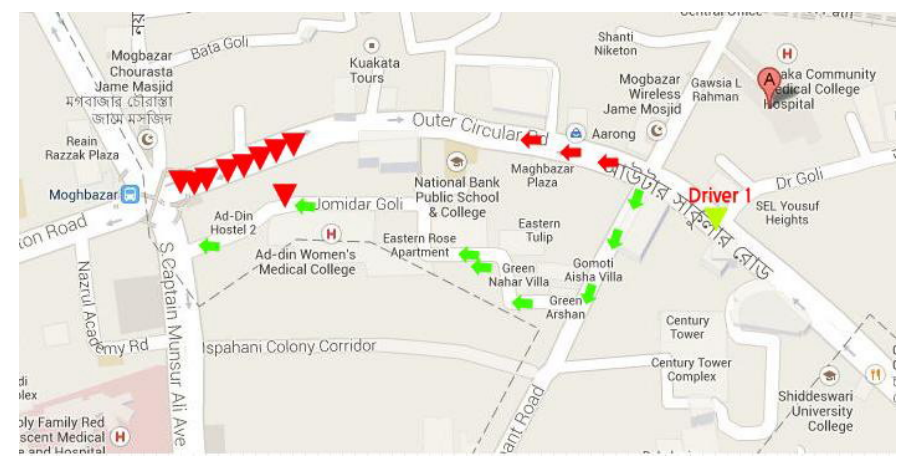

Fig. 8. View of Driver 1 upon entering "Moghbazar Circle" as destination.

route indicated on Fig. 9. This detour involves traversing an additional distance of 200 metres, and requires an additional estimated travel time of 1 minute.

Because of choosing the alternate route, the driver saves $6-1=5$ minutes by avoiding the "Moghbazar" traffic signal. A point to note is that the traffic signals at "Moghbazar" and "Bangla Motor" intersections turn Green simultaneously, and for 30 seconds after every 6-minute intervals as stated earlier. After crossing the "Moghbazar" intersection, the driver will still need the 7 minutes to travel the "Moghbazar" to "Bangla Motor" segment. As he had saved 5 minutes at the "Moghbazar" traffic signal and taken 7 minutes to reach "Bangla Motor" intersection, he actually reaches "Bangla Motor" $7-5=2$ minutes after the "Bangla Motor" traffic signal had last turned Green (recall that both signals turn Green simultaneously). So the driver hast to wait $6-2=4$ minutes for the "Bangla Motor" to turn Green again. Total estimated travel time using this alternate route is thus expected to be about $3+7+1+4=15$ minutes.

The new route involves a total distance of $2650+200=$ 2850 metres or 2.85 kilometres. Fuel consumption to cross (while moving) this distance will be $2.85 / 15=0.19$ cubic metre of CNG or 2.85/8 $=0.36$ litre of Octane. The additional cost for waiting in traffic in this scenario is $4 / 30=0.13$ cubic meter of CNG or $4 / 30=0.13$ litre of Octane. Total fuel consumption will thus be 0.32 cubic metre of CNG or 0.49 litre of Octane. At present rates of BDT 30 per cubic metre of CNG and BDT 98 per litre of Octane, the monetary costs of making this trip using the alternate route provided by RouteFinder evaluate to BDT 9.60 in case of CNG and BDT 48.02 in case of Octane.

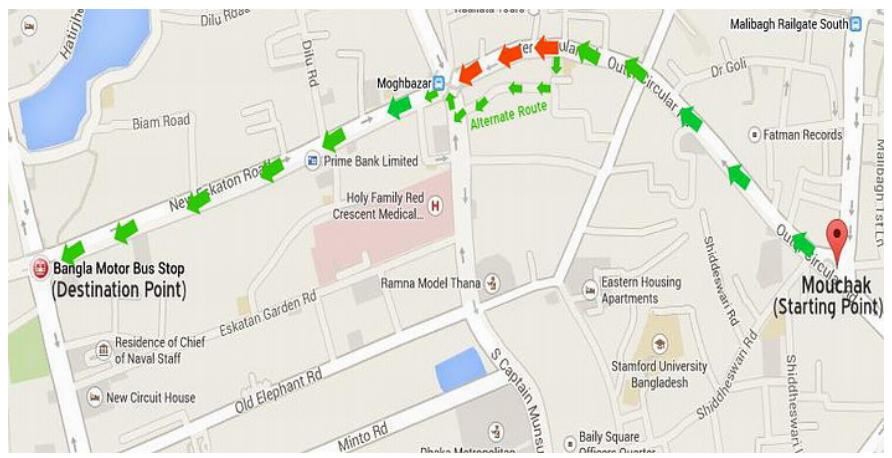

Fig. 9. Routes to go from "Mouchak" to "Bangla Motor".

The savings in travel time and fuel consumption achieved using RouteFinder are graphically illustrated in Figs. 10 and 11, respectively. The potential savings in both trip time and fuel consumption are obvious.

\section{CONCLUSION AND Future WORK}

Incessant traffic congestion is a perennial problem in many Asian metropolises. Most vehicle navigation systems use a Global Positioning System (GPS) to determine the location of the vehicle using satellite signals, and provide the shortest path route to a destination using pre-loaded digital maps. GPS navigation, however, typically calculates shortest path in terms of physical distance travelled. We know from experience that the shortest path in terms of distance may not always be the quickest at a particular time of day because of traffic congestions along the path. Developing real-time, optimum vehicle routing systems that take vehicle traffic density on the roads has thus recently become the focus of much research. To this end, we have developed "RouteFinder", a real-time, optimal vehicle routing system. RouteFinder is constructed using widely-available electronic parts, is inexpensive, provides an easy-to-use user interface on the driver's smartphone, and computes routes using signals from existing mobile phone networks. RouteFinder shows the locations of all vehicles in all possible routes from origin to destination, to enable the driver to choose the route with the minimum traffic density. This is in comparison to most similar recent proposals which either use expensive components, delay-centric and error-prone communication with satellites, or allow the tracking of the user's vehicle only.

The hardware module of RouteFinder contains the essential components of a cellular handset, formally known as a Mobile Station (MS), and a Subscriber Identity Module (SIM) and is powered by the vehicle's battery. As soon as the ignition switch is turned on, it establishes connection with the nearest Base Transceiver Station (BTS), and informs the driver of its online status through blinking Light Emitting Diodes (LEDs). The MS determines its location through a standard triangulation algorithm using signals from three nearest BTSs, and updates the mobile service provider's relevant Home Location Register (HLR) and Visitor Location Register (VLR) databases with the Mobile Stations International Mobile Subscriber Identity (IMSI) number [10]. The connection with the mobile operator's databases is performed using an Android $^{T M}$ application database. The database updates are done every few seconds. The main challenge here is to handle the long database 


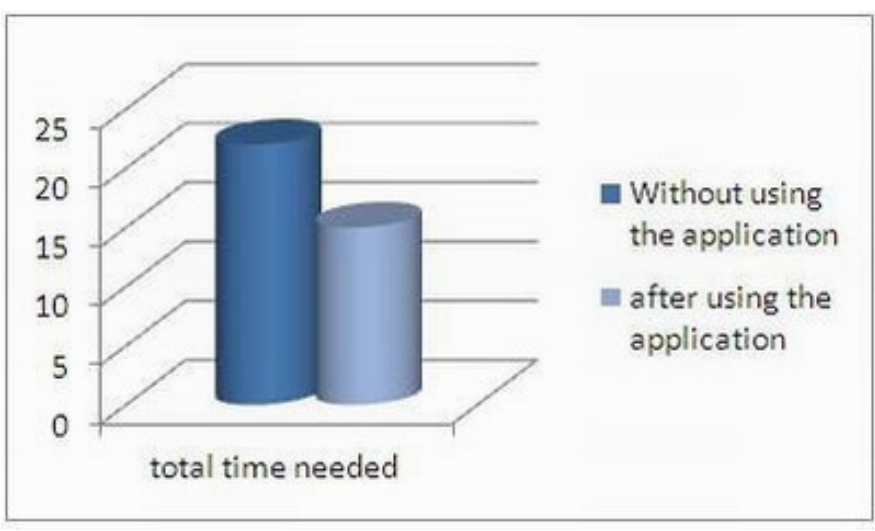

Fig. 10. Comparison of travel times with and without using RouteFinder.

queue of countless online vehicles and instantaneously refresh the connected MySQL database [13]. Finally, considering the popularity of smartphones and associated Android-based applications, we have developed an application for the driver's smartphone that will display in real-time the locations of all vehicles on all routes from the origin to the selected destination. The application fetches the data from the MySQL database and shows the locations of the vehicles on a Google $\mathrm{Map}^{T M}$, identifying them as moving or stationary by using Green or Red arrows, respectively. The driver thus knows which roads presently have a high density of vehicles on them and which do not. If every driver has this information about every road, then the total number of vehicles will eventually be naturally distributed among the different roads so that the likelihood of traffic buildup on any given road will be reduced. One must remember here that basic human nature is to follow conventional routes, and not veer off the beaten path.

We have implemented RouteFinder on 10 vehicles in Dhaka city, and presented sample results. Our calculations have demonstrated significant savings in not only time, but also in fuel consumptions.

Our future work will involve extending "RouteFinder" to mathematically determine the optimum route on the basis of four factors: total distance travelled, total time spent waiting for traffic signals at intermediate intersections to turn Green, number of number of vehicles on the roads and corresponding speed. We also wish to explore pattern matching algorithms to be able to predict upcoming traffic densities [16]-[19], in cases where estimated travel time for a selected origin-destination pair is large and traffic patterns in the given city is known to fluctuate rapidly.

\section{REFERENCES}

[1] "Number of Registered Motor Vehicles in Bangladesh (Yearwise)," Bangladesh Road Transport Authority (BRTA), Dhaka, Bangladesh, May 2014, statistical summary. Accessed: 01 Jun. 2014. [Online]. Available: http://www.brta.gov.bd/images/files/statistics/stat_bd_05-14. pdf

[2] Board of Investment. Organisation homepage. Accessed: 01 Jun. 2014. [Online]. Available: www.boi.gov.bd/index.php/about-bangladesh/ transportation-network\#road

[3] N. Yifeng, "Traffic congestion in bangladesh's capital causes nearly 2.86 bln usd a year," Beijing, China, 03 Aug. 2010, online newspaper article. Accessed: 01 Jun. 2014. [Online]. Available: http://english.people.com.cn/90001/90777/90851/7091782.html

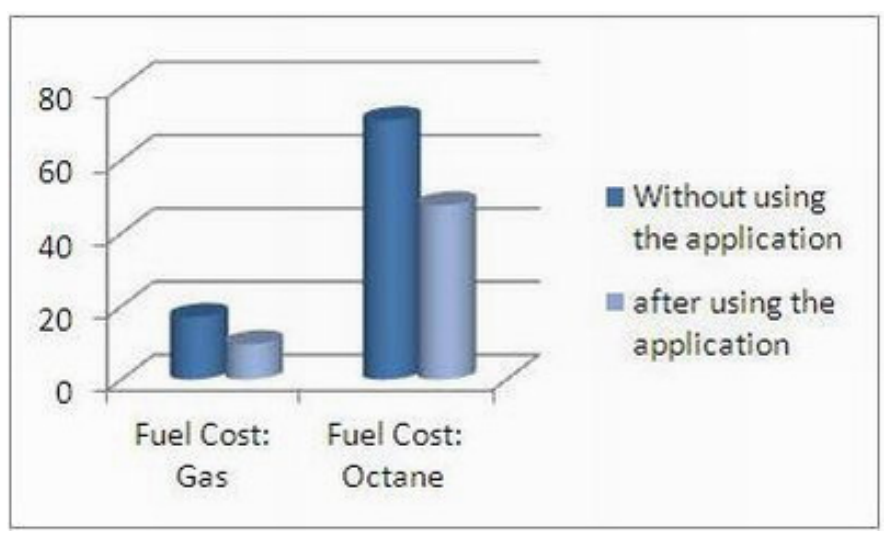

Fig. 11. Comparison of fuel cost estimates with and without using RouteFinder.

[4] SJ. Lee, G. Tewolde, and J. Kwon, "Design and implementation of vehicle tracking system using GPS/GSM/GPRS technology and smartphone application," in IEEE World Forum on Internet of Things (WF-IoT), Seoul, Korea Republic, Mar. 2014.

[5] V. Pierlot and M. V. Droogenbroeck, "A new three object triangulation algorithm for mobile robot positioning," IEEE Transactions on Robotics, vol. 30, no. 3, pp. 566-577, Jun. 2014.

[6] M. Fuad and M. Drieberg, "Remote vehicle tracking system using GSM Modem and Google map," in IEEE Int. Conf. on Sustainable Utilization and Development in Engineering and Technology (CSUDET), Selangor, Malaysia, Jun. 2013.

[7] M. Chaturvedi and S. Srivastava, "Real time vehicular traffic estimation using cellular infrastructure," in IEEE Int. Conf. on Advanced Networks and Telecommuncations Systems (ANTS), Kattankulathur, India, Dec. 2013.

[8] R. Gadri, B. Alhat, A. Chavan, S. Kamble, and R. Sonawane, "Land vehicle tracking system using Java on Android platform," Computer Engineering and Intelligent Systems, vol. 3, no. 5, pp. 88-94, Jul. 2012.

[9] D. Bajaj and N. Gupta, "GPS based automatic vehicle tracking using RFID," International Journal of Engineering and Innovative Technology, vol. 1, no. 1, pp. 31-35, Jan. 2012.

[10] T. Rappaport, Wireless Communications: Principles and Practice, 2nd ed. Upper Saddle River, NJ, USA: Prentice Hall, Jan. 2002.

[11] J. Kurose and K. Ross, Computer networking: A top-down approach, 5th ed. Boston, MA, USA: Addison Wesley, Mar. 2009.

[12] K. Olmstead, "Rethinking permissions in Android apps," Master's thesis, Georgetown University, Washington, DC, USA, May 2013.

[13] "MySQL 5.6 Reference Manual," Oracle Corp., Redwood Shores, CA, USA, 2014, technical manual. Accessed: 01 Jun. 2014. [Online]. Available: http://dev.mysql.com/doc/refman/5.6/en/index.html

[14] phpMyAdmin. Free software. Downloaded: 01 Jun. 2014. [Online]. Available: http://docs.phpmyadmin.net/en/latest/intro.html\# supported-features

[15] Jelly Bean. Software documentation. Accessed: 01 Jun. 2014. [Online]. Available: http://developer.android.com/about/versions/jelly-bean.html

[16] T. Ahmed, B. Oreshkin, and M. Coates, "Machine learning approaches to network anomaly detection," in Proc. ACM/USENIX Workshop on Tackling Computer Systems Problems with Machine Learning Techniques (SysML), Cambridge, MA, USA.

[17] T. Ahmed, M. Coates, and A. Lakhina, "Multivariate online anomaly detection using kernel recursive least squares," in Proc. IEEE Int. Conf. on Computer Communications (INFOCOM), Anchorage, AK, USA, May 2007.

[18] T. Ahmed, "Online anomaly detection using KDE," in Proc. IEEE Global Communications Conf. (GLOBECOM), Honolulu, HI, USA, Nov. 2009.

[19] — "Flow vector prediction using EM algorithms," in Proc. IEEE Int. Conf. on Communications (ICC), Cape Town, South Africa, May 2010. 